•综述・

\title{
异质生境中水生植物表型可塑性的研究进展
}

\author{
黎 否 $1,2,3$ 耿宇鹏 ${ }^{4}$ 兰志春 ${ }^{1,2,3}$ 陈家宽 ${ }^{1,2}$ 宋志平 $2^{*}$ \\ 1 (南昌大学生命科学研究院流域生态学研究所, 鄱阳湖环境与资源利用教育部重点实验室, 南昌 330031) \\ 2 (复旦大学生物多样性科学研究所, 上海 200438) \\ 3 (江西鄱阳湖湿地生态系统国家定位观测研究站, 南昌 330038) \\ 4 (云南大学生态学与地植物学研究所, 昆明 650091)
}

\begin{abstract}
摘要: 水生植物是一类以草本植物为主、与水紧密相关的生态类群, 大多数具有克隆性。面对水环境的变化, 水 生植物在形态、行为和生理上表现出多样化的表型可塑性, 对异质生境具有很强的适应能力。表型可塑性研究已 在陆生植物的多个类群展开, 然而目前对异质生境下水生植物的生态适应对策, 尤其是表型可塑性的研究尚重视 不够。本文在阐明克隆植物表型可塑性主要实现方式及其关系、水生环境异质性及其特点的基础上，重点从形态 可塑性、受食行为、克隆整合、克隆分工和风险分摊等 5 个方面讨论了水生植物如何通过表型可塑性适应异质性 水生环境。在今后的水生植物表型可塑性研究中，建议着重探讨以下问题：(1)表型可塑性的变化规律及机理; (2) 克隆整合对群落和生态系统的影响; (3)克隆整合与克隆片段化的权衡; (4)不同克隆构型的表型可塑性及其内在机 制; (5)表型可塑性的适应性进化; (6)水生植物与其他类群/营养级物种的关系; (7)水生生态系统对全球变化的响
\end{abstract} 应。

关键词: 环境异质性; 水生植物; 克隆生长; 形态可塑性; 克隆整合; 受食行为; 克隆分工; 风险分摊

\section{Phenotypic plasticity of aquatic plants in heterogeneous environments: a review}

Lei Li ${ }^{1,2,3}$, Yupeng Geng ${ }^{4}$, Zhichun Lan ${ }^{1,23}$, Jiakuan Chen ${ }^{1,2}$, Zhiping Song ${ }^{2 *}$

1 Center for Watershed Ecology, Institute of Life Science, Key Laboratory of Poyang Lake Environment and Resource Utilization, Ministry of Education, Nanchang University, Nanchang 330031

2 Institute of Biodiversity Science, Fudan University, Shanghai 200438

3 National Ecosystem Research Station of Jiangxi Poyang Lake Wetland, Nanchang 330038

4 Institute of Ecology and Geobotany, School of Life Sciences, Yunnan University, Kunming 650091

\begin{abstract}
Aquatic plants are the ecological group that mainly consists of herbaceous plants with habitats that are closely associated with water. Most aquatic plants have strong clonality. In response to changes in aquatic environments, aquatic plants exhibit significant plasticity in morphological, behavioral and physiological traits, and thus adapt well to heterogeneous aquatic environments. Compared with extensive studies on phenotypic plasticity of terrestrial plants, less attention has been paid to test how phenotypic plasticity of aquatic plants responds to heterogeneous environments. In this review, we briefly clarified the major types of phenotypic plasticity and their relationships in clonal plants in heterogeneous environments, identified the uniqueness of aquatic environments in relation to environmental heterogeneity, and analyzed the theoretical possibilities of aquatic plants showing high phenotypic plasticity. Furthermore, we probed into how aquatic plants adapted to heterogeneous aquatic environments by means of phenotypic plasticity involved with morphological plasticity, foraging behavior, clonal integration, intraclonal labour division, and risk spreading. Finally, we identified shortcomings in current studies on phenotypic plasticity of aquatic plants, and highlighted some issues deserving more attention in future studies, which include: (1) the changing pattern and mechanism of phenotypic plasticity; (2) the influence of clonal integration on community and ecosystem stability;
\end{abstract}

收稿日期: 2015-07-30; 接受日期: 2015-10-16

基金项目: 国家自然科学基金(31400403)、中国博士后科学基金(2015M571484)和国家林业局珍稀濒危物种野外救护与繁育项目(13007561)

* 通讯作者 Author for correspondence. E-mail: songzp@fudan.edu.cn 
(3) the trade-offs between clonal integration and fragmentation; (4) the differences of phenotypic plasticity in different types of clonal architecture, and their mechanisms; (5) the adaptive evolution of phenotypic plasticity; (6) the interaction of aquatic plants with species in other taxa or at different tropic levels; and (7) the response of aquatic ecosystems to global change.

Key words: environmental heterogeneity; aquatic plant; clonal growth; morphological plasticity; clonal integration; foraging behavior; intraclonal labour division; risk spreading

普遍存在的自然生境异质性使得植物获取资 源的难度大大增加(Jackson, 1993; Chen et al, 2002; 董鸣, 2011), 严重影响其生长、繁殖和扩散。表型 可塑性是植物适应异质生境的重要生态对策(Mal \& Lovett-Doust, 2005)。克隆植物具有克隆可塑性、 克隆整合和克隆分工等生活史性状, 对异质生境有 很强的适应能力(Herben, 2004; Roiloa et al, 2007; Pinno \& Wilson, 2014)。借助高度的表型可塑性, 在 异质生境中生长的克隆植物可以最大限度地获取 资源, 并在器官和组织间进行优化配置, 进而提高 资源的利用效率, 最终增加其适合度。对于那些遗 传多样性较低却占据多样化生境的物种(如许多水 生植物), 表型可塑性可能在其成功拓殖的过程中 起关键作用(Geng et al, 2007; Hyldgaard \& Brix, 2012; Riis et al, 2012)。

水生植物是指苔藓、蒝类及种子植物中, 植株 光合作用部分永久或周期性地浸没水中或浮于水 面(Cook, 1999)以适应水域环境的生态类群, 也包 括一些大型藻类 (如轮藻属 (Chara) 和丽藻属 (Nitella))(Cushing \& Allan, 2001; Chambers et al, 2008)。从严格意义上讲, 水生植物包括漂浮、浮叶、 沉水和挺水植物 4 大功能群 (Cronk \& Fennessy, 2001), 但广义上也将沼生植物与湿生植物包括在 内(Lacoul \& Freedman, 2006)。全球水生维管植物有 88 科 412 属 2,614 种, 约占所有维管植物物种数的 $1 \%$ (Chambers et al, 2008), 并且大部分是草本种类。陈 耀东等(2012)记录了我国的水生植物 61 科168属741 种(包括相近种 306种)。作为初级生产者, 水生植物 是水生生态系统健康运转的关键生物类群, 也是水 生生态系统多样性的基础。

克隆生长和无性繁殖在水生植物中非常普遍 (Grace, 1993), 因而很多水生植物的表型可塑性较 高, 能很好地适应异质生境。大量研究和观察表明, 水生植物的生存能力强, 能在高异质的生境中形成 单优群落。例如, 喜旱莲子草 (Alternanthera philoxeroides)可以适应不同水分条件的异质生境, 形成密集的毝炎状单优群落(Geng et al, 2007)。水生植 物还具有很强的种群扩展能力, 能够不断侵入邻近 群落, 扩大空间分布。例如, 凤眼莲(Eichnornia crassipes) 原产墨西哥, 因其旺盛的克隆生长而在世 界多个国家暴发成灾(Villamagna \& Murphy, 2010)。

水生植物的适应能力对高异质湿地和水生生 境的生物多样性维持与植被恢复起着重要的作用。 但是从全球范围来看, 入侵性水生杂草疯长会造成 生物多样性下降、物质循环失衡和水质恶化等后果 (Fleming \& Dibble, 2015), 并作为全球变化的重要 组成部分, 成为人类面临的严峻挑战。长期以来, 陆生植物的表型可塑性受到植物生态学界的持续 关注, 然而水生植物表型可塑性特别是其机制性研 究明显不足, 对现有研究也缺少系统性总结。本文 分析和梳理了水生植物(主要是克隆植物)表型可塑 性研究的主要进展, 分析了目前研究中存在的若干 问题, 并对如何推进该领域的研究进行了展望, 以 期引起更多研究者的关注。

\section{1 克隆植物表型可塑性的表现形式}

表型可塑性是指同一基因型由于环境条件的 改变在形态、生理和行为上作出相应变化的特性(de Kroon et al, 2005; Riis et al, 2010), 通常分为形态、 行为、生理和发育的可塑性(张大勇, 2000)。在异质 生境中, 为了最大限度地获取和利用生长与繁殖的 资源, 克隆植物一般采用形态结构的可塑性调节 (形态可塑性)、克隆分株选择性放置(受食行为)、分 株间物质传输与共享(克隆整合或生理整合)、相连 分株形态和生理等功能的特化(克隆分工)等策略 (Ikegami et al, 2008; 董鸣, 2011; Wang et al, 2011), 在某些情况下, 伴随克隆生长, 分株将有害物质(如 重金属离子、病原体等)分配到最适部位，从而将基 株死亡的风险分摊到各个独立的分株或分株系统 (风险分摊)(董鸣, 1996b; Gómez et al, 2008; Yan et 
al, 2013), 这有助于进一步提高其在异质生境中的 适合度。克隆植物表型可塑性的这 5 种主要表现形 式中, 受食行为主要借助克隆器官(如匍匐茎和根 茎)的形态可塑性完成; 克隆整合可以促进或削弱 形态可塑性, 也能诱发新的可塑性反应(de Kroon et al, 2005); 克隆分工在克隆整合的基础上, 通过分 株的形态和生理可塑性产生功能特化来实现; 而风 险分推的形式和机制与克隆整合是否存在及其强 度与发生时间紧密相关(朱志玲等, 2007)。

\section{2 水生环境的特殊异质性}

水生植物生长和繁殖的必需资源(光照、水分、 可溶性无机碳和矿质元素等)及其所处的环境条件 (干扰、采食、污染、沉积物掩埋、沉积物含氧量、 水位波动和地形等)并非均质, 而是呈现异质性分 布格局(Santamaría, 2002; Xiao et al, 2006), 这种异 质性在相对较小的尺度内(水文单元内或邻近水文 单元间)表现得更为明显(Santamaría, 2002)。

与陆生环境相比, 水生环境异质性的特殊性主 要表现在: (1)水生环境的光照强度不仅随水深在垂 直空间上呈现梯度变化, 还取决于浮游生物丰富 度、悬浮颗粒浓度和可溶性物质浓度(Holmes \& Klein, 1987; Chambers \& Prepas, 1988)。有些情况下 水平空间上的光环境也具有异质性。例如, “浑水态” 湖泊的水透明度在湖岸到湖心通常存在一个急剧 变化的梯度(Duarte et al, 1986); 在浮游植物、漂浮 植物、浮叶植物和沉积物等分布不均匀的水环境中, 光资源呈斑块状分布。(2)水生环境中可溶性无机碳 $\left(\mathrm{CO}_{2} 、 \mathrm{HCO}_{3}{ }^{-}\right.$或 $\left.\mathrm{CO}_{3}{ }^{2-}\right)$ 的浓度和形态一般会表现出 季节性和日变化(Maberly \& Spence, 1983; Santamaría, 1996), 在沉水植被斑块密集的浅水湖泊中, 碳的可利用性在水平空间上也常常有显著差异 (Spencer et al, 1994)。溶解氧(DO)、 $\mathrm{pH}$ 等也有类似 的变化, 且对水生环境的生物化学变化具有决定性 作用。(3)沉积物养分的空间异质性存在尺度差异, 它在微尺度内的差异与水中植被密度有关(Duarte \& Kalff, 1988), 在小尺度上的变化(如湖泊内部的 变化)一般与沉积物粒度有关(Santamaría, 2002), 而 在大尺度上的变化(如纬度差异)通常与不同气候对 沉积物矿化的影响有关。(4)河流、湖泊沿岸带沉积 物的颗粒组成和化学特征(有机物含量)均具有空间 异质性, 导致沉积物的含氧量在水平空间上产生差
异(Wetzel, 1988)。(5)水位波动是自然水体有别于陆 生环境的显著特征，水位波动的持续时间、幅度、 频率或淹水/退水时令和水流速度等方面的差异都 会增加环境异质性(Bornette \& Puijalon, 2011), 因此 长期的、季节性的水位波动会造成湿地的时空异质 性(Květ et al, 1988; Middleton et al, 2015; van der Valk et al, 2015)。例如，在急流环境中，水流速度在 空间上不一致, 处于植物斑块边缘的分株受到水流 冲击更大(Gantes \& Caro, 2001; Kotschy \& Rogers, 2008)。(6)虽然总体来讲, 水生环境温度的均质性较 高, 但在水生植被覆盖密集的浅水水域内, 水温通 常具有垂直方向的变化梯度, 这就导致植株温度在 垂直方向上发生较大变化(Santamaría, 2002)。

\section{3 水生植物具有较高表型可塑性}

由于水生环境的流通性与均匀分散性、水生生 物(如浮游植物)的游动性以及水下植物群落结构和 分层相对简单, 一般认为水生环境在小尺度的异质 性比陆生环境更低一些(Cook, 1985; Barrett et al, 1993), 空间异质性差异也更小。例如，河流、湖泊 等水生生态系统中土壤水分和温度在水平空间的 异质性显然要低于荒漠、盐碱化草地等陆地生态系 统; 水生植物群落冠层以下的光强变化也显然比森 林生态系统林冠内更小。理论上讲, 在小尺度上环 境均质性高的条件下, 植物表型可塑性的进化优势 会降低, 那么水生植物的表型可塑性是否会较低?

事实上, 大多数水生植物都具有较高的表型可 塑性。首先, 在水生环境中, 无性繁殖具有优势 (Grace, 1993), 这就使表型可塑性(而不是遗传分 化)的作用更重要。水生植物的繁育系统主要有以下 特点: (1)广泛而高效的克隆繁殖。很多证据表明, 多数水生植物都具有很强的克隆繁殖能力 (Grace, 1993; Philbrick \& Les, 1996), 其克隆繁殖发生的频 率远高于陆生植物(Santamaría, 2002), 常见的方式 有断枝(shoot fragment)、横生茎(creeping stem)如匍 匐茎和根茎、变态枝条基部(modified shoot base)如 鳞茎和球茎、根出条 (root sucker) 和假胎萌 (pseudovivipary)(Grace, 1993)。旺盛的克隆生长(特 别是多年生沉水和漂浮物种)形成遗传均一的种群。 (2)相对有限的有性繁殖。这既可能是过度克隆繁殖 的权衡造成的, 更有可能是由于水环境中有性繁殖 (开花、传粉、受精和结实)困难造成的。例如水位 
上升时, 大多数趋于产生气生花的物种的有性生殖 成功率降低(郭友好等, 1998; Santamaría, 2002), 还 有其他的极端环境(如水体污染、急流和风浪等引起 的机械损伤)也可能会减少有性繁殖的产生, 这就 限制了基因重组, 造成遗传多样性的降低。(3)克隆 繁殖和有性繁殖在环境因子作用下的权衡。很多研 究发现, 水生植物在环境因子影响下改变了资源分 配模式, 繁殖方式也发生了相应的改变(Thompson \& Eckert, 2004; van Drunen \& Dorken, 2012)。尽管 许多水生植物种群内的遗传变异很低(Zhang et al, 2010), 但是由于水生植物在广阔的地理范围内分 布(Chambers et al, 2008), 占据了多样的生境(Riis et al, 2010; Chappuis et al, 2014; Ganie et al, 2015), 其 较高的表型可塑性可以弥补遗传多样性低的不足, 适应多变的生境(耿宇鹏等, 2004)。

其次，克隆植物与非克隆植物相比，除了同一 个体上的以叶、芽为单位的有机体构件性以外, 还 具有以分株为单位的克隆构件性(董鸣, 1996a)。这 些构件在异质生境中的可塑性, 使得克隆植物比非 克隆植物具有更广泛的表型可塑性, 也有着更强的 环境适应能力(董鸣, 1996a, b)。Santamaría (2002)认 为水生植物在克隆生长方面表现出广泛的表型可 塑性, 其克隆生长习性、较强的局部扩散能力以及 水生环境中小尺度内的时空间异质性促进了表型 可塑性的进化。特别是对于在小空间和短时间尺度 上变化的因子, 水生植物的表型可塑性更加明显 (Santamaría, 2002)。

\section{4 水生植物表型可塑性的实验证据}

\section{1 形态可塑性}

形态可塑性是指不同环境条件和资源水平引 起的植物形态结构上的响应(de Kroons \& Hutchings, 1995)。克隆植物的形态可塑性主要表现为克 隆构型、资源吸收器官形态特征和生物量分配等方 面的变化, 是克隆植物适应异质生境的重要策略。

克隆植物的克隆构型可以分为密集型 (phalanx)、游击型(guerilla)及其过渡类型。密集型(间 隔子短、分枝少、分株分布较密集)有利于克隆植物 在异质生境中占据有利斑块, 提高对局部资源的利 用能力, 而游击型(间隔子长、分枝多、分株分布较 离散)有利于克隆植物逃离不利生境(Ye et al, 2006; Puijalon et al, 2008a)。
水生植物可以通过克隆构型的塑造来适应不 同的资源水平, 如凤眼莲的匍匐茎长度随营养水平 提高而增加(You et al, 2014c); 光异质生境(先端分 株遮阴而基端分株不遮阴) 中苦草 (Vallisneria natans) 的生物量和分株数大于光同质生境(先端分 株和基端分株均不遮阴)(Xiao et al, 2007)。

水生植物克隆构型还会随生境稳定性及环境 胁迫程度的变化而表现出可塑性。据报道, 生长在 美国 Tampa湾潮下浅滩的菜氏二药藻 (Halodule wrightii) 的形态对不同潮汐环境具有明显的适应, 同一斑块中不同位置的分株根茎节间长度依次为: 涨潮边缘>退潮边缘>斑块中部(Jensen \& Bell, 2001)。相对于静水生境, 沼生眼子菜(Potamogeton coloratus $)$ 的植株在流水生境中总匍匐茎和间隔子 更长(Puijalon et al, 2008a); 而在水流的作用下, 水 薄荷(Mentha aquatic)的根茎数增加(Puijalon et al., 2008a), 天山泽芹 (Berula erecta) 间隔子变短 (Puijalon \& Bornette, 2006), 可以形成更为密集的 冠层。还有研究表明, 在无 $\mathrm{Pb}$ 污染的环境中生长的 刺苦草(Vallisneria spinulosa)克隆体系分枝和分株 少, 趋于线型生长, 而异质 $\mathrm{Pb}$ 污染环境中产生的子 株匍匐茎长度显著增加, 逃离到无 $\mathrm{Pb}$ 生境的一级子 株产生较多的次级分株, 形成比较复杂的分枝状克 隆构型(严雪, 2003)。可见水生植物克隆构型对异质 生境的响应具有生态适应意义。

水陆梯度对水生植物形成了一种重要的环境 异质性。生长在土壤水分饱和-持续淹水连续环境 梯度上的植物会经历不同的淹水频率和淹水时长, 植株在不同生活史阶段经历的水文环境也可能大 不相同 (Well \& Pigliucci, 2000)。叶片异形性 (heterophylly) 是水生植物对水陆梯度的一种适应性 表型可塑性(adaptive phenotypic plasticity) (Wells \& Pigliucci, 2000)。Schmidt和Millington (1968)的研究 表明, 红雨伞草(Proserpinaca palustris)的主茎从下 到上生有沉水叶、浮水叶和气生叶, 以适应不同水 位的微生境; 北水苦荬(Veronica anagallis-aquatica) 在水流缓慢的条件下具有气生叶和沉水叶, 而在水 流速度高的条件下只具有沉水叶(Boeger \& Poulson, 2003)。De Wilde等(2014)通过控制实验发现, 天山泽芹、水茴草(Samolus valerandi)、水薄荷和北 水苦荬等植物的叶片在土壤暂时脱水的情况下, 叶 干物质含量增加而比叶面积减小, 产生了适应陆生 
生境的形态特征。

水生植物还可以通过调整克隆器官的形态和 生物量分配来适应水位变化。有研究发现, 深水中 的芦苇(Phragmites australis)根茎更短, 在基质中埋 深也更浅, 从而缩短了 $\mathrm{O}_{2}$ 的运输距离 (Weisner \& Strand, 1996; White \& Ganf, 2002)。当水位上升时, 水生植物能够将光合作用产物向地上器官转移, 使 得茎干或叶片伸长, 有利于增强对 $\mathrm{O}_{2} 、 \mathrm{CO}_{2}$ 和光资 源的利用, 同时减小根部生物量而减少呼吸消耗 (Visser et al, 2000; 罗文泊等, 2007)。如随着水位的 增加, 直立型沉水植物微齿眼子菜 (Potamogeton maackianus) 和竹叶眼子菜(P. malaianus)地下生物量 分配比例减小, 使得茎的生物量分配比例、株高和 比叶面积增加; 而莲座型的苦草会减少生物量向地 下部分的分配, 以增加叶生物量分配和株高(Fu et al, 2012); 穗状狐尾藻(Myriophyllum spicatum) 和绿 粉狐尾藻(M. aquaticum) 在水位上升的条件下分枝 长度增加而分枝数相应减少 (Strand \& Weisner, 2001; Hussner et al, 2009)。这些形态和生物量分配 的可塑性有利于植物更快地露出水面以增强其生 存能力。

除了简单的形态和生物量分配上的可塑性外, 在生物力学特征(如机械强度)方面的可塑性也是水 生植物适应水生环境(如水动力胁迫、水体富营养 化)的一种策略 (Puijalon et al, 2008b; Zhu et al, 2014)。例如, 与静水生境相比, 沼生眼子菜植株在 流水条件下通过提高水动力性能, 即减小弯曲角和 叶面阻力来缓冲水动力胁迫(Puijalon et al, 2008b)。

如上所述, 异质生境会促使水生植物采取一定 的形态可塑性对策, 并且其表现形式多种多样, 可 能以不同的机制影响植物生活史的不同阶段。因此 了解其机理有助于我们更清楚地认识水生植物适 应异质生境的机制以及形态可塑性在水生植物存 活、拓殖、种群建立、繁殖和扩散过程中的重要 意义。

\section{2 受食行为(分株选择性放置)}

在异质生境内, 为了增加对资源的获取, 克隆 植物通过改变间隔子长度、分枝强度和分枝角度等 形态学性状(van Kleunen \& Fischer, 2001), 将更多 分株有选择性地放置于资源相对丰富的小生境内, 被称为受食行为。受食行为使植物趋于规避资源贫 瘦的斑块并占据资源丰富的斑块 (de Kroons \&
Hutchings, 1995; van Kleunen \& Fischer, 2001)。间隔 子长度的可塑性具有很大的种间差异：随着资源水 平提高, 不同克隆植物的间隔子长度可能增加、变 短或不发生显著变化(de Kroons \& Hutchings, 1995); 克隆植物的分枝强度一般随资源水平的提 高而增大; 而分枝角度的可塑性很弱, 往往不对环 境变化产生响应(董鸣, 1996a)。

有关水生植物受食行为的研究目前并不多见。 对欧洲 Constance 湖的穿叶眼子菜 (Potamogeton perfoliatus)种群斑块的研究表明, $\mathrm{P}$ 含量高的斑块中 植株克隆分株数、分枝频率和平均分株长均显著大 于P含量低的斑块, 这说明穿叶眼子菜更倾向于将 资源吸收结构放置到资源较丰富的生境(Wolfer \& Straile, 2004)。在光资源异质生境中生长的苦草可以 有选择地将数量更多的叶片和分株分布到光资源 丰富的斑块中(Xiao et al, 2006; Zhao et al, 2013)。种 内和种间竞争也是塑造植物克隆器官的重要因素, 很多克隆植物能够通过间隔子的受食行为获取更 多资源, 进而在竞争中占优势(汤俊兵等, 2010)。Wu 等(2007)在克隆繁殖的荇菜(Nymphoides peltata) 与 以有性繁殖为主的菱 (Trapa bispinosa) 的实验中发 现, 当种间竞争发生时, 荇菜匍匐茎的匍匐茎长度 明显增加，可使其避开邻株干扰。

可见, 水生植物的受食行为对于其在水域环境 中的种群扩展、生境开拓和长期生存非常重要, 对 水生植物在自然界广泛分布具有一定的作用。

\section{3 克隆整合}

克隆整合是指相连分株通过间隔子或水平根 等横向结构沿源-汇梯度进行的光合产物、养分、 水分、次生产物和激素等物质的传输过程(Marshall, 1990; Stuefer et al, 2004), 是克隆植物区别于非克 隆植物的显著特征之一。分株间的克隆整合使生长 在资源较差斑块中或条件不利生境中(如水淹、动物 采食和重金属污染等)的分株可以间接利用其他较 好环境中分株的资源, 实现资源共享(董鸣, 2011)。 Marbà等(2002)采用同位素示踪法研究了C、N在8种 海草的母株与子株间的传输, 在所有物种的子株内 均检测到了施入母株的 ${ }^{13} \mathrm{C}$ 和 ${ }^{15} \mathrm{~N}$, 证实了分株间克 隆整合作用的存在, 证实克隆整合是海草分株在异 质生境中获取资源以及基株扩张与维持的重要 机制。

克隆整合强度对异质生境的资源分布状况有 
明显的可塑性反应。研究表明, 异质环境(与非遮荫 分株相连)中苦草分株的叶生物量和根生物量显著 高于同质环境(与遮荫分株相连), 说明遮荫分株可 以通过克隆整合从非遮荫分株获得物质补给(Xiao et al, 2007)。克隆整合对克隆植物的形态有一定的 修饰作用, 有助于增强水生植物对干旱、水淹、沉 积物掩埋和采食等环境胁迫的耐受能力。Touchette 等(2013)发现, 在干旱胁迫下的美洲水柳(Justicia americana $)$ 子株保持与水淹环境中母株的根茎相连 时, 其存活率和生长率都得到显著提高。喜早莲子 草的水淹处理实验表明, 水淹分株的生长和碳水化 合物积累可以通过克隆整合作用得到显著提高 (Luo et al, 2014)。另外, 克隆整合还可以显著缓解 沉积物掩埋对锯齿叶水丝草(Cymodocea serrulata) 和针叶藻(Syringodium isoetifolium)生长的不利影 响, 表现为在根茎连接的情况下, 掩埋深度大于 4 $\mathrm{cm}$ 的植株, 其地下部分(根、根茎) 生物量和枝条密 度都显著增加(Ooi et al, 2011)。You等(2014b)对喜 旱莲子草的研究发现, 与非去叶分株相连时, 去叶 分株的生长和光合速率(最大光化学量子产量 $F \mathrm{v} / F \mathrm{~m}$ 和非结构性碳水化合物含量)增加, 证明克隆 整合可以缓解动物取食对植株的胁迫。

克隆整合对于增强水生植物的入侵性(Yu et al, 2009; Song et al, 2013; You et al, 2014a)也有重要影 响。Elgersma等(2015)在同质园实验中发现, 水烛 (Typha angustifolia)、宽叶香蒲(Typha latifolia)及其 杂种粉绿香蒲 $($ Typha $\times$ glauca $) 3$ 个物种均存在母株 向子株的资源传递, 并且资源的传输量和传输距离 (即克隆整合强度)与不同物种的入侵性密切相关。 在喜早莲子草从陆地向水生生境扩张的模拟研究 中发现, 当其陆生生境内基端分株与水生生境内先 端分株的匍匐茎保持连接时, 其先端分株片段的生 长和克隆繁殖水平(总生物量、匍匐茎长度、分株数 和叶面积)显著提高, 这意味着克隆整合可以显著 地促进陆生生境中的喜旱莲子草种群向水域扩张 (Wang et al, 2009)。同样, Yu等(2009)通过模拟喜早 莲子草由裸地向草原植物群落的扩张过程, 证实克 隆整合促进了克隆分株对群落的入侵扩张, 但未改 变群落总生物量。

总之, 克隆整合使水生植物在异质生境中的无 性繁殖力和生存力更强, 有助于其完成定居与拓殖 过程。

\section{4 克隆分工}

为了更有效地获取地上和地下资源, 克隆植物 的相连分株伴随着克隆整合而产生形态与生理功 能的协调特化(Hutchings \& Wijesinghe, 1997; Wang et al, 2011), 称为克隆分工。实现克隆分工需具备两 个基本条件：分株间可通过克隆整合实现不同资源 的双向传递(资源共享)以及分株发生功能特化(董 鸣, 2011)。克隆分工是克隆植物有效利用异质性分 布资源的对策之一(Roiloa et al, 2007), 能给克隆植 物带来很多生存优势 (Peltzer, 2002; Peterson \& Chesson, 2002; Ikegami et al, 2008; You et al, 2014a)。

研究表明, 与非克隆植物个体相似, 在同质生 境中克隆植物个体的生物量分配对环境资源水平 的反应常常表现为“趋贫特化”(specialization for scarcity), 即将相对多的生物量投向吸收较缺乏资 源的器官(董鸣, 2011)。然而, 当克隆植物的相连分 株生长于由高资源斑块和低资源斑块组成的异质 环境条件下时, 克隆整合分株的生物量分配格局将 发生“趋富特化”(specialization for abundance), 即将 相对多的生物量投向吸收较丰富资源的器官 (Ikegami et al, 2008; 董鸣, 2011)。将相连的苦草分 株分别种植于同质和异质光照条件下, 发现光照充 足的分株叶生物量分配显著大于遮荫分株, 而根生 物量分配显著小于遮荫分株, 这表明分株对之间产 生了克隆分工(Xiao et al, 2007)。同样, 与高土壤营 养条件下的母株相连时, 喜旱莲子草子株的根生物 量分配显著小于与低土壤营养条件下的母株相连 的子株, 而其叶生物量分配显著增大, 说明根和叶 分别发生了利于土壤养分和光获取的功能特化 (You et al, 2014a)。

除了单一资源异质性环境, 水生生态系统中也 可能存在资源交互斑块性环境。例如, 滨海湿地红 树林(通过根瘤或根际固氮菌促进 $\mathrm{N}$ 的固定)或挺水 植物(通过促淤作用使 N、P沉积)冠层下的低光环境 可能是高养分斑块, 而远离这些植物的地方, 可能 光照资源充足而沉积物中养分的含量相对较低; 沉 水植物群落中部的冠层在形成低光环境的同时沉 积物养分含量可能相对丰富, 而其边缘的高光环境 下沉积物养分含量可能相对较低。很多研究都表明, 资源交互斑快性环境诱导的克隆分工有利于整个 基株对异质性环境资源的利用, 具有重要的生态适 
应意义(Stuefer et al, 1996; Liao et al, 2003; 董鸣, 2011)。但是相关的研究还很少, 需从水生植物生物 量分配的可塑性、资源吸收结构可塑性和生理可塑 性 3 个方面开展此类研究。克隆分工在水生植物生 长和繁殖过程中的作用还有待进一步探究。

\section{5 风险分摊}

伴随着克隆生长, 基株的死亡风险被分推到各 个分株或分株系统，降低了基株的死亡概率，从而 形成进化上的优势, 这种生态对策被称为风险分摊 (Cook, 1985; 董鸣等, 1996b)。不同的克隆植物由于 克隆生长型不同或所处的生境异质性不同, 可具有 不同的分摊形式和机制, 主要包括: 分株死亡概率 的独立性、克隆器官对资源的咜藏(Dong et al, 2010)、分株行为/表型的分化等3个方面(董鸣, 2011)。

国内外有关水生植物风险分摊对策的研究相 对较少。Yan等(2013)的研究结果表明, $\mathrm{Cu}$ 离子在苦 草的根中大量富集, 在向地上运输的同时在克隆体 系中实现向地下和水平方向扩散, 进而将污染胁迫 的风险分摊到整个克隆种群。生长于异质性 $\mathrm{Pb}$ 污染 环境中的苦草将母株吸收的一部分有害重金属 $\mathrm{Pb}$ 通过匍匐茎向生长在健康生境中的子株转移, 在源 株和子株体内产生有害的活性氧和自由基, 形成风 险分推，有助于克隆体系的保持(Yan et al, 2006)。 另有研究表明, 风险分摊可能有利于克隆植物从感 染病原体的土壤中逃离(D'Hertefeldt \& van der Putten, 1998)。

水生植物的枝条在外界环境扰动(如水流速度、 洪水、鱼类取食和人类活动等)影响下容易形成断枝 (克隆片段化)(Dong et al, 2010)。断枝是水生植物进 行无性繁殖的重要方式, 不仅繁殖代价低, 而且有 利于促进远交和长距离扩散(Santamaría, 2002)。克 隆片段的长度、位置、是否有叶等特征都对分株的 存活和生长有不同程度的影响(Dong et al, 2010; 周 洁等, 2012)。只要水生植物的克隆片段化不影响甚 至能提高基株的适合度, 就可以体现其在风险分摊 上的作用。但是克隆片段化究竟是否会对基株的表 现和适合度有影响, 以及影响是正面还是负面的, 目前仍无定论。

风险分摊的过程同时也是克隆植物适应环境 和利用资源的过程。目前只对极少数的水生植物进 行过相关研究, 关于不同克隆构型或环境因素导致
水生植物采取不同风险分摊对策的机制，还需在不 同生境中对更多植物开展研究，才能得出普适性的 结论。

\section{5 存在的问题及展望}

长期以来, 植物的表型可塑性一直受到生态学 家的普遍关注。水生植物因其独特的进化过程、生 活史特征和生理适应、多样化的繁殖模式以及在浅 水湖泊中的关键建构功能而引起越来越多国际学 者的关注。然而, 与陆生植物相比, 现有的水生植 物的表型可塑性研究仍显薄弱。主要表现在: (1)目 前的大多数研究局限在淡水生态系统, 而滨海和海 洋生态系统的植物研究较少; (2)水生植物表型可塑 性的表现形式多种多样, 但有关水生植物受食行 为、克隆分工和风险分摊的研究还很少，且很少深 入探讨水生植物形态、行为和生理可塑性变化的机 制; (3)对水生植物表型可塑性的研究主要集中于对 单个物种或近缘种间的比较上，较少涉及克隆可塑 性、克隆整合和克隆分工等在群落水平上的作用; (4)定量地表达克隆构型的工作较少，对于不同克隆 构型(游击型和密集型、根茎型和匍匐茎型)水生植 物的表型可塑性特征的比较研究十分缺乏。

由于水生植物表型可塑性的研究与生态学许 多前沿领域(如生物多样性、生物入侵、生物进化和 全球变化等)相关，因此未来应加强以下方面的研 究:

(1)水生环境水平方向异质性的量化。目前有关 水生环境垂直方向上异质性的量化研究已经有很 多，如水深对光照的影响(Chambers \& Prepas, 1988; Middelboe \& Markager, 1997); 然而水平方向上异 质性的量化研究还很薄弱, 如光照斑块、沉积物养 分的异质性等。克隆植物对环境异质性的响应理论 目前更多地体现在水平空间的小尺度异质性上。

(2)水生植物表型可塑性特征的变化规律及机 理。目前对水生植物克隆整合的方向、速率和强度 等格局，以及环境胁迫对原有克隆整合格局的修饰 作用知之甚少; 克隆分工形成的条件和途径以及环 境条件在此过程中的诱导作用仍无定论; 各类克隆 构型的水生植物在不同环境中的风险分摊形式和 机制也尚不清楚; 克隆分工和风险分摊是否在不同 克隆构型水生植物中广泛存在, 还有待进一步验 证。此外, 环境对水生植物不同水平等级(分株水 
平、分株系统水平和基株水平)表型变化的影响以及 各等级水平之间的相互作用, 也值得重点研究。

(3)克隆整合对群落和生态系统的影响。有关克 隆整合对群落物种组成和多样性以及对生态系统 结构、过程和功能的影响研究非常薄弱 (Wilsey, 2002; Yu et al, 2010), 而有关水生植物克隆整合在 群落中作用的研究更加缺乏(Li et al, 2015)。外来水 生植物借助克隆整合的优势迅速入侵本地水生植 物群落已成为全球性的生态问题, 这方面的研究有 利于客观评估外来水生植物入侵造成的影响。

(4)克隆整合与克隆片段化的权衡。对水生植物 而言, 由于分株能随水流移动, 因此在广阔的水面 上(如大型河流、溪流或湖泊), 克隆片段化是非常有 利于扩散的(Okada et al, 2009; Dong et al, 2012)。在 相对均质的水生环境中, 克隆整合对整个克隆的表 现不一定有显著贡献(Wang et al, 2009; Gao et al, 2013; Wang et al, 2014), 而克隆片段化可能更有 利。这方面的研究也有待进一步加强。

(5)不同克隆构型水生植物的表型可塑性特征 及其内在机制。水生植物各类克隆构型的表型可塑 性是与生境的资源分布状况相关还是由其在系统 进化过程中形成的克隆构型决定, 需进行不同克隆 类型和不同生境的对比实验, 对克隆植物的适应性 假说和限制性假说作进一步检验。

(6)水生植物表型可塑性的适应性进化。表型可 塑性是物种适应性进化的一个重要方面, 但并不是 所有的表型可塑性变异都是适应性的, 许多性状的 变异可能是因为有机体的生长在资源受限条件下 受到胁迫的结果, 是被动的反应(van Kleunen \& Fischer, 2005)。对于分布广、适应性强的水生植物 而言, 其适应性进化研究如多基因型比较研究目前 并不多见(van Kleunen et al, 2000; van Kleunen \& Fischer, 2001), 许多性状的可塑性究竟是适应性的 还是被动的反应还不清楚, 有待进一步探讨。

(7)水生植物与群落中其他类群/营养级的物种 (如浮游植物、底栖动物、鱼类和水鸟等)相互作用 下的表型可塑性。对这些相互作用对表型可塑性的 影响研究, 将有助于认识水生植物物种多样性的形 成和维持机制, 而群落水平的研究可以在一个复杂 而自然的生态背景下全面地考虑植物对生物环境 与非生物环境的适应与进化。

(8)水生生态系统与全球变化。水生生态系统对
全球变化较为敏感, 全球变化带来的水体富营养 化、水体污染、水位波动、气候变暖等威胁着水生 植物的生物多样性, 例如, 湖泊富营养化和全球变 暖都有利于外来水生植物的生长与无性繁殖(You et al, 2014c), 增强其入侵性。因此，探讨环境变化、 水生植物表型可塑性和生态系统功能三者之间的 关系, 亦是值得生态学者们长期关注的问题。

\section{参考文献}

Barrett SCH, Echert CG, Husband BC (1993) Evolutionary processes in aquatic plant populations. Aquatic Botany, 44, 105-145.

Boeger MRT, Poulson ME (2003) Morphological adaptations and photosynthetic rates of amphibious Veronica anagallis-aquatica L. (Scrophulariaceae) under different flow regimes. Aquatic Botany, 75, 123-135.

Bornette G, Puijalon S (2011) Response of aquatic plants to abiotic factors: a review. Aquatic Sciences, 73, 1-14.

Chambers PA, Lacoul P, Murphy KJ, Thomaz SM (2008) Global diversity of aquatic macrophytes in freshwater. Hydrobiologia, 595, 9-26.

Chambers PA, Prepas EE (1988) Underwater spectral attenuation and its effect on the maximum depth of angiosperm colonization. Canadian Journal of Fish Aquatic Science, 45, 1010-1017.

Chappuis E, Gacia E, Ballesteros E (2014) Environmental factors explaining the distribution and diversity of vascular aquatic macrophytes in a highly heterogeneous Mediterranean region. Aquatic Botany, 113, 72-82.

Chen YD (2011) The Chinese Aquatic Plants. Henan Science and Technology Press, Zhengzhou. (in Chinese) [陈耀东 (2011) 中国水生植物. 河南科学技术出版社, 郑州.]

Chen YF, Yu FH, Dong M (2002) Scale-dependent spatial heterogeneity of vegetation in $\mathrm{Mu}$ Us sandy land in semi-arid area of China. Plant Ecology, 162, 135-142.

Cook CDK (1999) The number and kinds of embryo-bearing plants which have become aquatic. Perspectives in Plant Ecology, Evolution and Systematics, 2, 79-102.

Cook RE (1985) Growth and development in clonal plant population. In: Population Biology and Evolution of Clonal Organism (ed Jackson JBC, Buss LW, Cook RE), pp. 259-296. Yale University Press, New Haven.

Cronk JK, Fennessy MS (2001) Wetland Plants: Biology and Ecology. CRC Press, Florida.

Cushing CE, Allan JD (2001) Streams: Their Ecology and Life. Academic Press, San Diego.

de Kroons H, Huber H, Stuefer JF, van Groenendael JM (2005) A modular concept of phenotypic plasticity in plants. New Phytologist, 166, 73-82.

de Kroons H, Hutchings MJ (1995) Morphological plasticity in clonal plants: the foraging concept reconsidered. Journal of Ecology, 83, 143-152.

De Wilde M, Sebei N, Puijalon S, Bornette G (2014) Re- 
sponses of macrophytes to dewatering: effects of phylogeny and phenotypic plasticity on species performance. Evolutionary Ecology, 2014, 28, 1155-1167.

D'Hertefeldt T, van der Putten WH (1998) Physiological integration of the clonal plant Carex arenaria and its response to soil-borne pathogens. Oikos, 81, 229-237.

Dong BC, Alpert P, Guo W, Yu FH (2012) Effects of fragmentation on the survival and growth of the invasive, clonal plant Alternanthera philoxeroides. Biological Invasions, 14, 1101-1110.

Dong BC, Yu GL, Guo W, Zhang MX, Dong M, Yu FH (2010) How internode length, position and presence of leaves affect survival and growth of Alternanthera philoxeroides after fragmentation? Evolutionary Ecology, 24, 1447-1461.

Dong M (1996a) Clonal growth in plants in relation to resource heterogeneity: foraging behavior. Acta Botanica Sinica, 38, 828-835. (in Chinese with English abstract) [董鸣 (1996a) 资源异质环境中的植物克隆生长: 受食行为. 植物学报, 38, 828-835.]

Dong M (1996b) Plant clonal growth in heterogeneous habitats: risk-spreading. Acta Phytoecologica Sinica, 20, 543-548. (in Chinese with English abstract) [董鸣 (1996b) 异质性生境 中的植物克隆生长: 风险分摊. 植物生态学报, 20, 543-548.]

Dong M (2011) Clonal Plant Ecology. Science Press, Beijing. (in Chinese) [董鸣 (2011) 克隆植物生态学. 科学出版社, 北京.]

Duarte CM, Kalff J (1988) Influence of lake morphometry on the response of submerged macrophytes to sediment fertilization. Canadian Journal of Fish Aquatic Science, 45, 216-221.

Duarte CM, Kalff J, Peters RH (1986) Patterns in biomass and cover of aquatic macrophytes in lakes. Canadian Journal of Fish Aquatic Science, 43, 1900-1908.

Elgersma KJ, Wildová R, Martina JP, Currie WS, Goldberg DE (2015) Does clonal resource translocation relate to invasiveness of Typha taxa? Results from a common garden experiment. Aquatic Botany, 126, 48-53.

Fleming JP, Dibble ED (2015) Ecological mechanisms of invasion success in aquatic macrophytes. Hydrobiologia, 746, 23-37.

Fu H, Yuan GX, Cao T, Ni LY, Zhang M, Wang SR (2012) An alternative mechanism for shade adaptation: implication of allometric responses of three submersed macrophytes to water depth. Ecological Research, 27, 1087-1094.

Ganie AH, Reshi ZA, Wafai BA, Puijalon S (2015) Phenotypic plasticity: cause of the successful spread of the genus Potamogeton in the Kashmir Himalaya. Aquatic Botany, 120, 283-289.

Gantes HP, Caro AS (2001) Environmental heterogeneity and spatial distribution of macrophytes in plain streams. Aquatic Botany, 70, 225-236.

Gao L, Li B, Liu WY, Shen YX, Liu WJ (2013) Inhibition effects of daughter ramets on parent of clonal plant Eichhornia crassipes. Aquatic Botany, 107, 47-53.
Geng YP, Pan XY, Xu CY, Zhang WJ, Li B, Chen JK, Lu BR, Song ZP (2007) Phenotypic plasticity rather than locally adapted ecotypes allows the invasive alligator weed to colonize a wide range of habitats. Biological Invasions, 9, 245-256.

Geng YP, Zhang WJ, Li B, Chen JK (2004) Phenotypic plasticity and invasiveness of alien plants. Biodiversity Science, 12, 447-455. (in Chinese with English abstract) [耿宇 鹏, 张文驹, 李博, 陈家宽 (2004) 表型可塑性与外来植 物的入侵能力. 生物多样性, 12, 447-455.]

Gómez S, Onoda Y, Ossipov V, Stuefer JF (2008) Systemic induced resistance: a risk-spreading strategy in clonal plant networks? New Phytologist, 179, 1142-1153.

Grace JB (1993) The adaptive significance of clonal reproduction in angiosperms: an aquatic perspective. Aquatic Botany, 44, 159-180.

Guo YH, Huang SQ, Chen JK (1998) Breeding system and evolution of aquatic angiosperms. Acta Hydrobiologica Sinica, 22, 79-85. (in Chinese with English abstract) [郭友 好, 黄双全, 陈家宽 (1998) 水生被子植物的繁育系统与 进化. 水生生物学报, 22, 79-85.]

Herben T (2004) Physiological integration affects growth form and competitive ability in clonal plants. Evolutionary Ecology, 18, 493-520.

Holmes MG, Klein WH (1987) The light and temperature environments. In: Plant Life in Aquatic and Amphibious Habitats (ed. Crawford RMM), pp. 3-22. Blackwell, Oxford.

Hussner A, Meyer C, Busch J (2009) The influence of water level and nutrient availability on growth and root system development of Myriophyllum aquaticum. Weed Research, 49, 73-80.

Hutchings MJ, Wijesinghe DK (1997) Patchy habitats, division of labor and growth dividends in clonal plants. Trends in Ecology \& Evolution, 12, 390-394.

Hyldgaard B, Brix H (2012) Intraspecies differences in phenotypic plasticity: invasive versus non-invasive populations of Ceratophyllum demersum. Aquatic Botany, 97, 49-56.

Ikegami M, van Hal S, van Rheenen JW, Whigham DF, Werger MJ (2008) Spatial division of labour of Schoenoplectus americanus. Plant Ecology, 199, 55-64.

Jackson RB, Caldwell MM (1993) The scale of nutrient heterogeneity around individual plants and its quantification with geostatistics. Ecology, 74, 612-614.

Jensen S, Bell S (2001) Seagrass growth and patch dynamics: cross-scale morphological plasticity. Plant Ecology, 155, 201-217.

Kotschy K, Rogers K (2008) Reed clonal characteristics and response to disturbance in a semi-arid river. Aquatic Botany, 88, 47-56.

Květ J, Westlake S, Dykyjová D, Marshall EJP, Ondok JP (1988) Primary production in wetlands. In: The Production Ecology of Wetlands: The IBP Synthesis (ed Westlake DF, Květ J, Szczepański A), pp. 78-169. Cambridge University Press, Cambridge.

Lacoul P, Freedman B (2006) Environmental influences on 
aquatic plants in freshwater ecosystems. Environmental Reviews, 14, 89-136.

Li HL, Xu YS, Wang YY, Yu NQ, Zhang MX, Lei GC, Yu FH (2015) Does clonal fragmentation of the floating plant Eichhornia crassipes affect the growth of submerged macrophyte communities?. Folia Geobotanica, 2015, 50, 283-291.

Liao MJ, Yu FH, Song MH, Zhang SM, Zhang JZ, Dong M (2003) Plasticity in R/S ratio, morphology and fitnessrelated traits in response to reciprocal patchiness of light and nutrients in the stoloniferous herb, Glechoma longituba. Acta Oecologica, 24, 231-239.

Luo FL, Chen Y, Huang L, Wang A, Zhang MX, Yu FH (2014) Shifting effects of physiological integration on performance of a clonal plant during submergence and de-submergence. Annals of Botany, 113, 1265-1274.

Luo WB, Xie YH, Song FB (2007) Survival strategies of wetland plants in flooding environments. Chinese Journal of Ecology, 26, 1478-1485. (in Chinese with English abstract) [罗文泊, 谢永宏, 宋风斌 (2007) 洪水条件下湿地植物 的生存策略. 生态学杂志, 26, 1478-1485.]

Maberly SC, Spence DHN (1983) Photosynthetic inorganic carbon use by freshwater plants. Journal of Ecology, 71, 705-724.

Mal TK, Lovett-Doust J (2005) Phenotypic plasticity in vegetative and reproductive traits in an invasive weed, Lythrum salicaria (Lythraceae), in response to soil moisture. American Journal of Botany, 92, 819-825.

Marbà N, Hemminga MA, Mateo MA, Duarte CM, Maas YEM, Terrados J, Gacia E (2002) Carbon and nitrogen translocation between seagrass ramets. Marine Ecology Progress Series, 226, 287-300.

Marshall C (1990) Source-sink relations of interconnecte ramets. In: Clonal Growth in Plants: Regulations and Functions (eds van Groenendael J, de Kroon H), pp. 23-41. SPB Academic Publishing, Hague.

Middelboe AL, Markager S (1997) Depth limits and minimum light requirements of freshwater macrophytes. Freshwater Biology, 37, 553-568.

Middleton BA, van der Valk AG, Davis CB (2015) Responses to water depth and clipping of twenty-three plant species in an Indian monsoonal wetland. Aquatic Botany, 126, 38-47.

Okada M, Grewell BJ, Jasieniuk M (2009) Clonal spread of invasive Ludwigia hexapetala and L. grandiflora in freshwater wetlands of California. Aquatic Botany, 91, 123-129.

Ooi JLS, Kendrick GA, van Niel KP (2011) Effects of sediment burial on tropical ruderal seagrasses are moderated by clonal integration. Continental Shelf Research, 31, 1945-1954.

Peltzer DA (2002) Does clonal integration improve competitive ability? A test using aspen (Populus tremuloides [Salicaceae]) invasion into prairie. American Journal of Botany, 89, 494-499.

Peterson AG, Chesson P (2002) Short-term fitness benefits of physiological integration in the clonal herb Hydrocotyle pe- duncularis. Austral Ecology, 27, 647-657.

Philbrick CT, Les DH (1996) Evolution of aquatic angiosperm reproductive systems: What is the balance between sexual and asexual reproduction in aquatic angiosperms? BioScience, 46, 813-826.

Pinno BD, Wilson SD (2014) Nitrogen translocation between clonal mother and daughter trees at a grassland-forest boundary. Plant Ecology, 215, 347-354.

Puijalon S, Bornette G (2006) Phenotypic plasticity and mechanical stress: biomass partitioning and clonal growth of an aquatic plant species. American Journal of Botany, 93, 1090-1099.

Puijalon S, Bouma TJ, Van Groenendael J, Bornette G (2008a) Clonal plasticity of aquatic plant species submitted to mechanical stress: escape versus resistance strategy. Annals of Botany, 102, 989-996.

Puijalon S, Léna JP, Rivière N, Champagne JY, Rostan JC, Bornette G (2008b) Phenotypic plasticity in response to mechanical stress: hydrodynamic performance and fitness of four aquatic plant species. New Phytologist, 177, 907-917.

Riis T, Lambertini C, Olesen B, Clayton JS, Brix H, Sorrell BK (2010) Invasion strategies in clonal aquatic plants: Are phenotypic differences caused by phenotypic plasticity or local adaptation? Annals of Botany, 106, 813-822.

Riis T, Olesen B, Clayton JS, Lambertini C, Brix H, Sorrell BK (2012) Growth and morphology in relation to temperature and light availability during the establishment of three invasive aquatic plant species. Aquatic Botany, 102, 56-64.

Roiloa SR, Alpert P, Tharayil N, Hancock G, Bhowmik PC (2007) Greater capacity for division of labour in clones of Fragaria chiloensis from patchier habitats. Journal of Ecology, 95, 397-405.

Santamaría L (2002) Why are most aquatic plants widely distributed? Dispersal, clonal growth and small-scale heterogeneity in a stressful environment. Acta Oecologica, 23, 137-154.

Santamaría L, Montes C, Hootsmans MJM (1996) Influence of environmental parameters on the biomass development of Ruppia drepanensis populations in Doñana National Park: the importance of conditions affecting the underwater light climate. International Journal of Salt Lake Research, 5, 157-180.

Schmidt BL, Millington WF (1968) Regulation of leaf shape in Proserpinaca palustris. Bulletin of the Torrey Botanical Club, 95, 264-286.

Song YB, Yu FH, Keser LH, Dawson W, Fischer M, Dong M, van Kleunen M (2013) United we stand, divided we fall: a meta-analysis of experiments on clonal integration and its relationship to invasiveness. Oecologia, 171, 317-327.

Spencer WE, Teeri J, Wetzel RG (1994) Acclimation of photosynthetic phenotype to environmental heterogeneity. Ecology, 75, 301-314.

Strand JA, Weisner SE (2001) Morphological plastic responses to water depth and wave exposure in an aquatic plant (Myriophyllum spicatum). Journal of Ecology, 89, 166-175. 
Stuefer JF, de Kroon H, During HJ (1996) Exploitation of environmental heterogeneity by spatial division of labour in a clonal plant. Functional Ecology, 10, 328-334.

Stuefer JF, Gómez S, van Mölken T (2004) Clonal integration beyond resource sharing: implications for defence signalling and disease transmission in clonal plant networks. Evolutionary Ecology, 18, 647-667.

Tang JB, Xiao Y, An SQ (2010) Advance of studies on rhizomatous clonal plant ecology. Acta Ecologica Sinica, 30, 3028-3036. (in Chinese with English abstract) [汤俊兵, 肖 燕, 安树青 (2010) 根茎克隆植物生态学研究进展. 生态 学报, 30, 3028-3036.]

Thompson FL, Eckert CG (2004) Trade-offs between sexual and clonal reproduction in an aquatic plant: experimental manipulations vs. phenotypic correlations. Journal of Evolutionary Biology, 17, 581-592.

Touchette BW, Moody JW, Byrne CM, Marcus SE (2013) Water integration in the clonal emergent hydrophyte, Justicia americana: benefits of acropetal water transfer from mother to daughter ramets. Hydrobiologia, 702, 83-94.

van der Valk AG, Volin JC, Wetzel PR (2015) Predicted changes in interannual water-level fluctuations due to climate change and its implications for the vegetation of the Florida Everglades. Environmental Management, 55, 799-806.

van Drunen WE, Dorken ME (2012) Trade-offs between clonal and sexual reproduction in Sagittaria latifolia (Alismataceae) scale up to affect the fitness of entire clones. New Phytologist, 196, 606-616.

van Kleunen M, Fischer M (2001) Adaptive evolution of plastic foraging responses in a clonal plant. Ecology, 82, 3309-3319.

van Kleunen M, Fischer M, Schmid B (2000) Clonal integration in Ranunculus reptans: by-product or adaptation? Journal of Evolutionary Biology, 13, 237-248.

van Kleunen M, Fischer M (2005) Constraints on the evolution of adaptive phenotypic plasticity in plants. New Phytologist, 166, 49-60.

Villamagna AM, Murphy BR (2010) Ecological and socio-economic impacts of invasive water hyacinth (Eichhornia crassipes): a review. Freshwater Biology, 55, 282-298.

Visser EJW, Bögemann GM, van de Steeg HM, Pierik R, Blom CWPM (2000) Flooding tolerance of Carex species in relation to field distribution and aerenchyma formation. New Phytologist, 148, 93-103.

Wang P, Xu YS, Dong BC, Xue W, Yu FH (2014) Effects of clonal fragmentation on intraspecific competition of a stoloniferous floating plant. Plant Biology, 16, 1121-1126.

Wang N, Yu FH, Li PX, He WM, Liu J, Yu GL, Song YB, Dong M (2009) Clonal integration supports the expansion from terrestrial to aquatic environments of the amphibious stoloniferous herb Alternanthera philoxeroides. Plant Biology, 11, 483-489.

Wang Z, Li Y, During HJ, Li L (2011) Do clonal plants show greater division of labour morphologically and physiologi- cally at higher patch contrasts? PLoS ONE, 6, e25401.

Weisner SE, Strand JA (1996) Rhizome architecture in Phragmites australis in relation to water depth: implications for within-plant oxygen transport distances. Folia Geobotanica, 31, 91-97.

Wells CL, Pigliucci M (2000) Adaptive phenotypic plasticity: the case of heterophylly in aquatic plants. Perspectives in Plant Ecology, Evolution and Systematics, 3, 1-18.

Wetzel RG (1988) Water as an environment for plant life. In: Vegetation of Inland Waters (ed. Symoens JJ), pp. 1-30. Kluwer Academic Publishers, Dordrecht.

White S, Ganf GG (2002) A comparison of the morphology, gas space anatomy and potential for internal aeration in Phragmites australis under variable and static water regimes. Aquatic Botany, 73, 115-127.

Wilsey B (2002) Clonal plants in a spatially heterogeneous environment: effects of integration on Serengeti grassland response to defoliation and urine-hits from grazing mammals. Plant Ecology, 159, 15-22.

Wolfer SR, Straile D (2004) Spatio-temporal dynamics and plasticity of clonal architecture in Potamogeton perfoliatus. Aquatic Botany, 78, 307-318.

Wu ZH, Yu D, Tu MH, Wang Q, Xiong W (2007) Interference between two floating-leaved aquatic plants: Nymphoides peltata and Trapa bispinosa. Aquatic Botany, 86, 316-320.

Xiao KY, Yu D, Wang JW (2006) Habitat selection in spatially heterogeneous environments: a test of foraging behaviour in the clonal submerged macrophyte Vallisneria spiralis. Freshwater Biology, 51, 1552-1559.

Xiao KY, Yu D, Xu XW, Xiong W (2007) Benefits of clonal integration between interconnected ramets of Vallisneria spiralis in heterogeneous light environments. Aquatic Botany, 86, 76-82.

Yan X (2003) Study of Ecological Response of Aquatic Plants to Stress. PhD dissertation, Wuhan University, Wuhan. (in Chinese with English abstract) [严雪 (2003) 水生植物的 逆境生态学研究. 博士学位论文, 武汉大学, 武汉.]

Yan X, Wang HW, Wang QF, Rudstam LG (2013) Risk spreading, habitat selection and division of biomass in a submerged clonal plant: responses to heterogeneous copper pollution. Environmental Pollution, 174, 114-120.

Yan X, Yu D, Wang HY, Wang JW (2006) Response of submerged plant (Vallisneria spinulosa) clones to lead stress in the heterogenous soil. Chemosphere, 63, 1459-1465.

Ye XH, Yu FH, Dong M (2006) A trade-off between guerrilla and phalanx growth forms in Leymus secalinus under different nutrient supplies. Annals of Botany, 98, 187-191.

You WH, Fan SF, Yu D, Xie D, Liu CH (2014a) An invasive clonal plant benefits from clonal integration more than a co-occurring native plant in nutrient-patchy and competitive environments. PLoS ONE, 9, e97246.

You WH, Yu D, Xie D, Han C, Liu C (2014b) The invasive plant Alternanthera philoxeroides benefits from clonal integration in response to defoliation. Flora, 209, 666-673.

You WH, Yu D, Xie D, Yu LF, Xiong W, Han CM (2014c) 
Responses of the invasive aquatic plant water hyacinth to altered nutrient levels under experimental warming in China. Aquatic Botany, 119, 51-56.

Yu FH, Wang N, He WM, Dong M (2010) Effects of clonal integration on species composition and biomass of sand dune communities. Journal of Arid Environments, 74, 632-637.

Yu FH, Wang N, Alpert P, He WM, Dong M (2009) Physiological integration in an introduced, invasive plant increases its spread into experimental communities and modifies their structure. American Journal of Botany, 96, 1983-1989.

Zhang DY (2000) Researches on Theoretical Ecology. Higher Education Press, Beijing. (in Chinese) [张大勇 (2000) 理 论生态学研究. 高等教育出版社, 北京.]

Zhang YY, Zhang DY, Barrett S (2010) Genetic uniformity characterizes the invasive spread of water hyacinth. Molecular Ecology, 19, 1774-1786.

Zhao CF, Li HL, Luo FL (2013) Effects of light heterogeneity on growth of a submerged clonal macrophyte. Plant Species Biology, 28, 156-164.
Zhou J, Wang D (2012) Survival strategies of stem fragments in narrow endemic and widespread plants of the aquatic genus Myriophyllum. Acta Hydrobiologica Sinica, 36, 316-322. (in Chinese with English abstract) [周洁, 王东 (2012) 狐尾藻属狭域种和广域种断枝的生长与再生能力 比较研究. 水生生物学报, 36, 316-322.]

Zhu GR, Cao T, Zhang M, Ni LY, Zhang XL (2014) Fertile sediment and ammonium enrichment decrease the growth and biomechanical strength of submersed macrophyte Myriophyllum spicatum in an experiment. Hydrobiologia, 727, 109-120.

Zhu ZL, Li DZ, Wang XP, Sheng LJ, Shi Q (2007) Water physiology integration and its ecological effect of clonal plants. Acta Botanica Boreali-Occidentalia Sinica, 26, 2602-2614. (in Chinese with English abstract) [朱志玲, 李 德志, 王绪平, 盛丽娟, 石强 (2007) 克隆植物的水分生 理整合及其生态效应. 西北植物学报, 26, 2602-2614.]

(责任编委: 于飞海 责任编辑: 时意专) 